\title{
Análise da influência da morfologia do modelo ósseo na distribuição das tensões do sistema de implante cone morse, método dos elementos finitos tridimensionais
}

\author{
Analysis of the influence of bone model morphology on stress distribution of \\ the Morse taper implant system, method of three-dimensional finite elements
}

Rander Avelar

Aline Serrado de Pinho Barcellos ${ }^{* *}$

Ronaldo Luís Carvalho***

Estevão Tomomitsu Kimpara ${ }^{* * * *}$

\section{Resumo}

O conhecimento do comportamento mecânico do sistema de implante corresponde a um importante parâmetro no prognóstico de uma reabilitação protética. Objetivo: avaliar a influência do formato de tecido ósseo na distribuição de tensões no implante do tipo cone morse e tecido ósseo por meio do método dos elementos finitos tridimensionais. Materiais e método: foram utilizados três tipos de formatos de modelos para tecidos ósseos: a) paralelepípedo; b) tronco elíptico; c) gerado a partir dos dados de uma tomografia. Nos modelos tridimensionais, foram instalados implantes que receberam aplicação de duas cargas de $100 \mathrm{~N}$ no sentido craniocaudal: 1) na direção vertical ao longo do eixo axial e 2) na direção oblíqua a $45^{\circ}$ do eixo axial de vestibular para lingual. Resultados: as tensões de von Mises máximas $(\mathrm{MPa})$ nos modelos do implante cone morse (CM) e do tecido ósseo do tipo paralelepípedo foram de 14.30 no carregamento vertical e de 60.78 no carregamento oblíquo; no modelo de tecido ósseo elíptico, foram de 14.69 no carregamento vertical e de 71.66 no carregamento oblíquo; no modelo de tecido ósseo com a configuração originada da tomografia, foram de 15.04 no carregamento vertical e de 79.15 no carregamento oblíquo. Conclusão: o formato dos modelos de tecido ósseo influenciou no comportamento e na distribuição das tensões, sendo que o modelo gerado pelos dados da tomografia apresentou os maiores valores de tensões.

Palavras-chave: Análise de elementos finitos. Implantes dentários. Mecânica.

\section{Introdução}

Os trabalhos científicos de Branemark e colaboradores $^{1,}$ que descreveram o fenômeno da osseointegração, trouxeram grandes avanços na realização do tratamento de pacientes desdentados, alterando de maneira significativa o planejamento para a reposição da perda dentária. Dessa maneira, a terapia com implantes apresenta atualmente um alto índice de sucesso, superando $90 \%$ para as situações de implantação em mandíbula ${ }^{2-4}$. Apesar da expec-

Doutor em Odontologia pelo Instituto de Ciência e Tecnologia de São José dos Campos da Universidade Estadual Paulista, São José dos Campos, São Paulo, Brasil.

Mestranda do Programa de Pós-Graduação em Odontologia Restauradora do Instituto de Ciência e Tecnologia de São José dos Campos da Universidade Estadual Paulista, São José dos Campos, São Paulo, Brasil.

Doutorando do Programa de Pós-Graduação em Odontologia Restauradora do Instituto de Ciência e Tecnologia de São José dos Campos da Universidade Estadual Paulista, São José dos Campos, São Paulo, Brasil.

**** Doutor em Odontologia. Professor titular de Materiais Dentários do Departamento de Materiais e Prótese do Instituto de Ciência e Tecnologia de São José dos Campos da Universidade Estadual Paulista, São José dos Campos, São Paulo, Brasil. 
tativa de resultados positivos, existem fatores que podem influenciar no desempenho do tratamento com implantes. A transmissão da carga pelo implante e a distribuição das tensões no tecido ósseo representam fatores significativos na determinação do sucesso ou da falha do sistema ${ }^{5-9}$.

A análise do comportamento mecânico, no que diz respeito às distribuições de tensões em um sistema de reabilitação por implantes, pode ser realizada por fotoelasticidade, extensometria e pelo método dos elementos finitos ${ }^{10-12}$, além dos clássicos métodos analíticos para sistemas estruturais simples. Contudo, a avaliação, quando envolve geometrias complexas, torna difícil a determinação da solução analítica, sendo que o método dos elementos finitos utiliza procedimentos computacionais numéricos que auxiliam na compreensão do comportamento das tensões ${ }^{6,13-15}$.

O método dos elementos finitos é uma técnica que permite solucionar um problema complexo dividindo-o em domínios simples e pequenos, chamados elementos finitos, combinando suas equações lineares locais adequadamente para prover a solução de todo o conjunto ${ }^{3,6,11,13,14,16}$. Realizando análises em que os objetos de estudo estão representados de acordo com a realidade, de tal forma que o tecido ósseo pode ser concebido como um sólido simples, como, por exemplo, um paralelepípedo ${ }^{17,18}$, um tronco elíptico ${ }^{3,9,10,13,14,19}$, ou gerado com riqueza de detalhes esculturais utilizando-se de recursos imaginológicos ${ }^{3,4,20}$.

O nível de fidelidade do objeto a ser analisado com as suas características físicas reais podem vir a influenciar nos resultados. Modelos mais realísticos da geometria complexa e detalhada, como do tecido ósseo, podem ser gerados com auxílio de técnicas avançadas de imagem digital. No entanto, modelos minuciosos aumentam os requisitos necessários para a análise e, consequentemente, também aumentam o tempo e os recursos necessários para o processamento $^{3,4}$.

O presente estudo teve por objetivo avaliar a compreensão das limitações da ferramenta de análise pelo método dos elementos finitos bem como o quanto a sofisticação dos modelos se faz necessária para a obtenção de resultados coerentes com os fenômenos em estudo, o que contribui para o uso racional do método. Visto que o modelo pode vir a influenciar nos resultados, surgiu a necessidade de se avaliar o efeito da modelagem óssea na distribuição de tensões no implante e desse para o tecido de suporte, utilizando o método dos elementos finitos tridimensionais.

\section{Materiais e método}

O método dos elementos finitos, empregado na avaliação proposta, constou das seguintes etapas: a) seleção e construção do modelo a ser testado, tecido ósseo simulando mandíbula humana com densidade D1, segundo classificação de Lekholm e Zarb ${ }^{21}$ (1985); b) modelo de implante com conexão protética do tipo cone morse, com pilar protético; c) determinação e construção do ambiente do carregamento, restrições e condições para a análise; d) solução.

Os objetos estudados foram modelados geometricamente, subdivididos em elementos interconectados por pontos nodais formando um conjunto denominado de malha, à qual se atribuem propriedades mecânicas dos materiais em estudo. $\mathrm{O}$ ambiente foi definido, estabelecendo-se as condições de vínculo ou de restrição e carregamento para a análise dos resultados em função da solicitação imposta. Os resultados foram expressos utilizando-se o critério de tensões equivalentes, também conhecidas como tensões de von Mises.

\section{Aquisição inicial das imagens para análise}

O tecido ósseo da região mandibular de um indivíduo foi digitalizado por meio da tomografia computadorizada de feixe cônico (TCFC) e, posteriormente, segmentado no programa de Mimics (Materialise, Bélgica), gerando arquivos armazenados em mídia digital compact disc (CD). O exame da tomografia foi realizado conforme o protocolo no qual a aquisição das imagens ocorreu, no plano axial, cortes de $1 \mathrm{~mm}$ e espaçamento de $0,5 \mathrm{~mm}$ entre cada corte, sendo que o ângulo do cabeçote do equipamento, conhecido também como Gantry Tilt, foi $0^{\circ}$.

Associando-se à mandíbula para a simulação proposta, foi selecionado o implante cônico com conexão do tipo cone morse (CM), com 8,5 mm de comprimento na parte da espira e $1,8 \mathrm{~mm}$ correspondente à região do pescoço (Titanium Fix, A.S. Technology, Brasil), conectado ao pilar protético personalizável para prótese cimentada. As imagens bidimensionais e as peças foram utilizadas na geração da imagem volumétrica obtida pela modelagem tridimensional no programa SolidWorks (SolidWorks, EUA).

\section{Pré-processamento}

Por meio do programa Mimics (Materialise, Bélgica), realizou-se um corte na mandíbula, obtendo-se uma fatia da mandíbula envolvendo a linha média, tendo as dimensões aproximadas de $13,8 \mathrm{~mm}$ no sentido laterolateral (mésio-distal) da borda superior, e $13,65 \mathrm{~mm}$ na inferior, na borda distal $24,6 \mathrm{~mm}$ de altura e na borda mesial $21,85 \mathrm{~mm}$ de altura, 8,5 $\mathrm{mm}$ no sentido vestíbulo-lingual e $2 \mathrm{~mm}$ de largura. O arquivo no formato STEP foi importado para o programa SolidWorks (SolidWorks, EUA), que integrou o modelo da mandíbula, gerado na digitalização pelo tomógrafo e processado pelo programa Mimics (Materialise, Bélgica), com os implantes e pilares protéticos, modelados pelo mesmo programa. Os mo- 
delos do tecido ósseo no formato de paralelepípedo e tronco elíptico foram, também, gerados no programa SolidWorks, apresentando no modelo do tipo paralelepípedo as dimensões de $12,75 \mathrm{~mm}$ na direção horizontal e sentido laterolateral, $23 \mathrm{~mm}$ na direção vertical e sentido craniocaudal, e 9,6 mm na direção horizontal e sentido anteroposterior. O modelo tipo tronco elíptico apresentou, na direção vertical e sentido craniocaudal, $23,5 \mathrm{~mm}$, na direção horizontal e sentido laterolateral, $14,1 \mathrm{~mm}$ e, na direção horizontal e sentido anteroposterior, $10,54 \mathrm{~mm}$, na região correspondente ao centro e na parte correspondente ao início do raio de fechamento da parte superior e inferior, $6,98 \mathrm{~mm}$. Os modelos de tecido ósseo/sistema de implante foram importados para o programa de análise de elementos finitos Ansys Workbench 9.0 (Ansys, EUA), módulo Desing Modeler, realizando, dessa forma, a integração dos objetos modelados e/ou importados no programa SolidWorks para Ansys. O módulo Simulation do programa Ansys define e aplica as condições do ambiente de simulação, no qual é gerada a malha contendo os elementos e nós para o cálculo dos deslocamentos pelo programa.

\section{Processamento e análise da simulação}

O programa Ansys Workbench 9.0, módulo Simulation, realizou a simulação da aplicação de uma carga de $100 \mathrm{~N}$ no eixo axial e outra aplicação de $100 \mathrm{~N}$ a $45^{\circ}$ desse eixo, no sentido de vestibular para lingual, no implante inserido no segmento ósseo no formato de paralelepípedo, tronco elíptico e gerado pela tomografia computadorizada.

\section{Condições da interface osso/implante}

Os modelos dos implantes foram considerados conectados por meio de nós comuns ao modelo de osso ao longo de toda a interface, simulando uma condição de osseointegração ideal, ou seja, $100 \%$ de contato ósseo, bem como o contato dos pilares protéticos com os implantes, que foram considerados monobloco implante/pilar protético.

\section{Elementos tetraédricos sólidos e nós usados para malha}

Os modelos simulando o tecido ósseo, a saber: paralelepípedo, denominado de A; tronco elíptico, B e o originado a partir da tomografia computadoriza$\mathrm{da}, \mathrm{C}$, bem como o modelo de implante cone morse foram discretizados, ou seja, subdivididos em volumes justapostos, em uma malha de elementos conectados por nós apresentando um volume de 142,75 $\mathrm{mm}^{3}, 14103$ elementos e 23087 nós. O modelo A, ao ser preparado para receber o implante CM, apresentou um volume de $2732,9 \mathrm{~mm}^{3}$, 18121 elementos e 27592 nós. O tipo $\mathrm{B}$, ao receber o implante $\mathrm{CM}$, teve um volume de $2731,83 \mathrm{~mm}^{3}$, 19404 elementos e 29318 nós. O modelo $\mathrm{C}$ apresentou um volume de $2733,13 \mathrm{~mm}^{3}$, 218754 elementos e 308037 nós.

\section{Condições do contorno}

A malha construída com elementos de transição com hexaedro e elevado alisamento, associado ao controle da malha e a um teste de $10 \%$ de convergência, determina o número total de elementos e nós. Os vínculos impostos aos nós, estabelecendo os pontos de fixação, formaram as restrições aos deslocamentos e às rotações, em uma condição de engaste perfeito, sendo aplicados às bordas laterais correspondentes à mesial e à distal dos modelos que representavam o tecido ósseo ${ }^{17}$.

\section{Solução}

O critério escolhido para análise das tensões foi o de von Mises, conhecido como critério de máxima energia de deformação ou de tensões equivalentes, obtido a partir da raiz quadrada da soma dos quadrados, produzindo sempre resultados positivos. Não se pode aferir se o mapa de tensões representa tensões de tração, compressão ou cisalhamento. As tensões de von Mises ( $\mathrm{MPa}$ ) foram registradas e analisadas quanto à magnitude e à distribuição no implante e no tecido ósseo.

\section{Resultados}

Os resultados quantitativos das tensões originadas nos implantes e modelos de tecido ósseo são apresentados nas Figuras 1 e 2. No aspecto quantitativo, apresentam-se valores em $\mathrm{N} / \mathrm{mm}^{2}(\mathrm{MPa})$, e no qualitativo, pelo gradiente de cores correspondentes às tensões máximas e mínimas.

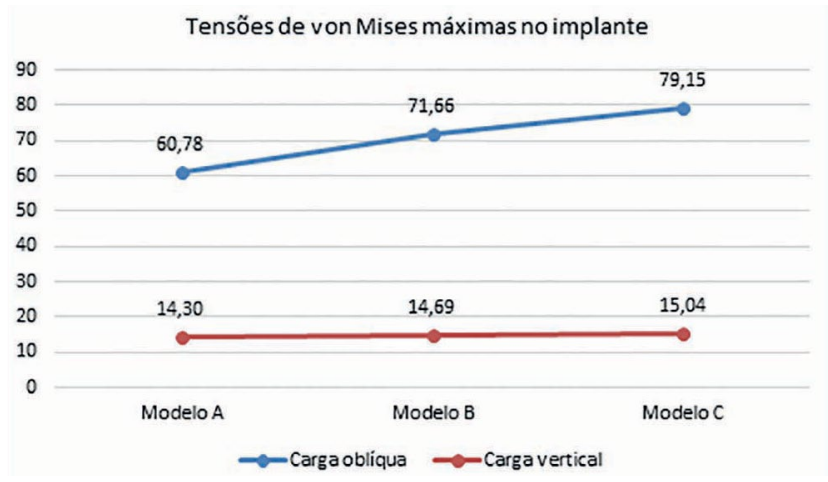

Figura 1 - Comportamento do implante nos diferentes modelos de tecido ósseo, valores expressos em MPa

Tensões de von Mises (MPa) máximas no implante sob carga vertical e oblíqua. Fonte: dados da pesquisa. 


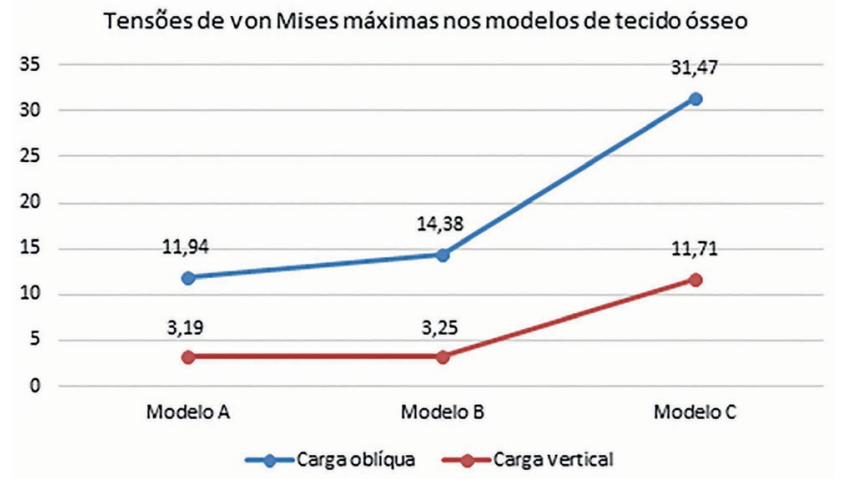

Figura 2 - Comportamento dos diferentes modelos de tecido ósseo, valores expressos em $\mathrm{MPa}$

Tensões de von Mises máximas nos modelos de tecido ósseo sob carga vertical e oblíqua.

Fonte: dados da pesquisa

\section{Discussão}

O método dos elementos finitos tem sido extensivamente utilizado como ferramenta de análise mecânica na odontologia ${ }^{9,12-14,22-25}$, porém, como qualquer técnica de pesquisa, apresenta limitações inerentes ao método, às simplificações impostas ao ambiente da análise e ao modelo ${ }^{6}$. A interpretação dos resultados quantitativos apresenta a distribuição de forças dentro do implante. No entanto, a interpretação da área externa do implante é questionável, em virtude de que a modelagem do ambiente em que foi realizada a análise pode influenciar nos resultados, como observado por Weinstein ${ }^{26}$ (1976), que, ao modelar a condição de superfície porosa do implante, obteve resultados mais próximos aos do laboratório. Verificou-se, também, pelos resultados do presente estudo, que o tipo de modelo ósseo pode influenciar nos valores obtidos. Adicionalmente, os resultados qualitativos suportam comparações que permitem conclusões a respeito do objeto em estudo ${ }^{27}$.

O método dos elementos finitos correlaciona-se diretamente com a qualidade dos modelos nos quais se realiza a simulação. A geometria dos objetos estudados, como o tecido ósseo de suporte, em especial, e os implantes, apresenta aspecto não paramétrico. A simplificação dos modelos pode levar a resultados não acurados ${ }^{6}$. Observa-se que os trabalhos de pesquisas têm modelado o tecido ósseo e o implante por meio de diversas formas e estratégias. Estudos mostraram que diferentes comportamentos das tensões dos modelos foram observados pelos autores em uma situação semelhante de carregamento, mostrando que simplificações de modelos devem ser consideradas com cautela ${ }^{3,4,13,14,17,18,28}$.

A qualidade de tecido ósseo tem importante papel na osseointegração bem como no comportamento mecânico. Implantes instalados em osso tipo IV apresentam uma probabilidade maior de falha quando comparados às fixações inseridas em ossos tipo I, II e III ${ }^{21,29}$. É importante a presença de osso de boa qualidade na região do pescoço do implante para suportar a concentração de tensões nessa regiã $0^{30}$, pois a qualidade e a quantidade de osso estão diretamente correlacionadas ao sucesso dos implantes osseointegrados ${ }^{31}$. Por esse motivo, o modelo ósseo adotado neste trabalho foi considerado do tipo I, no qual há uma camada homogênea de cortical óssea, possibilitando a avaliação das distribuições de tensões em diferentes modelos ósseos dentro de uma qualidade ideal e indicada para instalação de implantes. Uma vez que, em estudo posterior ${ }^{19}$, já foi demostrado que modelos ósseos do tipo I, avaliados por meio do método de elementos finitos, apresentam menor micromovimentação e, por consequência, uma diminuição das intensidades das tensões concentradas nas regiões que sofrem carregamento.

A avaliação das cargas que podem ocorrer clinicamente pode auxiliar na determinação das condições a serem empregadas em trabalhos laboratoriais. As forças utilizadas neste estudo foram retiradas de estudos clínicos de forças mastigatórias. Foi encontrada como força máxima durante o fechamento da mandíbula, em pacientes reabilitados com implantes e prótese do tipo protocolo de Branemark, uma intensidade média de $186,6 \mathrm{~N}$ para o homem e $115,9 \mathrm{~N}$ para a mulher, tendo como média para a mastigação o valor de 50,1 $\mathrm{N}^{32,33}$. Outros estudos encontraram como valor médio para a mastigação $166 \mathrm{~N}^{15}$, forças verticais de $60 \mathrm{~N}$ a $150 \mathrm{~N}^{34}$. Portanto, nota-se que in vivo há uma grande variabilidade em função dos aspectos individuais dos pacientes.

De modo semelhante, os trabalhos de pesquisas com implantes apresentam uma grande diversidade no que diz respeito à magnitude da carga aplicada, existindo uma amplitude de $50 \mathrm{~N}$ a $300 \mathrm{~N}$ de força e uma carga de $100 \mathrm{~N}^{4,12,23,25,27,35-38}$. Observando a magnitude de força aplicada pelos trabalhos, ponderando pesquisas anteriore $\mathrm{s}^{33,39} \mathrm{e}$ considerando que o presente trabalho comparou diferentes formatos de modelos, optou-se pela carga de $100 \mathrm{~N}$, aplicada ao pilar protético, na região de contato com a coroa protética, na direção vertical e a $45^{\circ}$, como foi adotado em outros trabalhos ${ }^{23,35}$. Cargas oblíquas têm o potencial efeito de propiciar concentração de tensões no terço cervical bem como solicitar mais da estrutura sob carregamento, devendo ser considerado o momento de força no planejamento da prótese e evitada a sobrecarga, com vistas à longevidade do tratamento com implantes ${ }^{7}$, tornando-se, dessa forma, imprescindível na simulação de cargas oblíquas ${ }^{24}$.

Os resultados deste estudo evidenciaram as mais altas tensões quando da aplicação de cargas oblíquas, tanto para o implante quanto para o tecido ósseo, conforme observado, também, por Papavisilou $^{40}$ (1997). As maiores tensões no tecido ósseo localizaram-se no osso marginal ${ }^{19,41}$, adverte-se quanto ao efeito acumulativo quando ocorre coincidência espacial das tensões máximas durante o carregamento vertical e oblíquo, que poderiam levar à reabsorção óssea induzida em virtude das tensões. 
O modelo influenciou diretamente nos resultados encontrados para as tensões equivalentes no tecido ósseo, sendo que os maiores valores ocorreram no modelo tipo $\mathrm{C}$, gerado a partir da tomografia computadorizada. Observa-se que, à medida que o modelo de tecido ósseo aproxima-se da forma real da mandíbula, forma escultural, há um incremento nos valores encontrados, tanto na carga vertical quanto na oblíqua. O modelo A e B apresentaram tensões de von Mises correspondentes a, aproximadamente, $27 \%$ dos valores do modelo $\mathrm{C}$, para a carga vertical, enquanto que, para a carga oblíqua, as tensões correspondem a aproximadamente $40 \%$.

Holmes e Loftus ${ }^{19}$ (1997) modelaram um segmento de mandíbula no formato de tronco elíptico, aplicaram uma carga de $100 \mathrm{~N}$ a $30^{\circ}$ na coroa metálica, em ouro, conectada ao pilar protético, e este ao implante tipo parafuso com $3,75 \mathrm{~mm} \mathrm{X} 10$ $\mathrm{mm}$ de comprimento. Foram encontradas tensões equivalentes, para o osso tipo I, na magnitude de 13,7 $\mathrm{MPa}$, valores próximos aos encontrados para o modelo tronco elíptico, tipo $\mathrm{B}$, avaliado neste trabalho, que foi de 14,38 $\mathrm{MPa}$. A pequena diferença entre os valores poderia ser creditada às dimensões do implante e ao tecido ósseo, ligeiramente distintos entre os estudos, ao ângulo de aplicação da carga e ao módulo de elasticidade.

As tensões encontradas nos implantes foram semelhantes na carga vertical, variando em torno de $10 \%$. Na carga oblíqua, houve uma maior variabilidade, alternando entre $60,78 \mathrm{MPa}$, para o implante no modelo $\mathrm{A}$, e 79,15 $\mathrm{MPa}$, para o implante inserido no modelo $\mathrm{C}$.

Em situações nas quais se necessita analisar o comportamento de novos desenhos frente à resposta mecânica, os modelos simplificados utilizados na análise de elementos finitos podem auxiliar, de forma rápida e com menor custo, na seleção do modelo e prosseguir no desenvolvimento dos estudos. Quando o objetivo é correlacionar os aspectos mecânicos com a resposta biológica, o modelo próximo ao padrão físico real pode representar melhor os fenômenos que estão ocorrendo. Pesquisas adicionais poderão tornar possível o planejamento individualizado com menor possibilidade de falhas, visto que as variáveis biológicas do hospedeiro podem modificar a resposta.

\section{Conclusão}

Com base nos resultados observados, pode-se concluir que o modelo ósseo 3D com maior nível de detalhamento e com formato mais próximo do real possibilita uma avaliação mais correta das magnitudes das tensões resultantes do carregamento de implantes instalados nessas estruturas.

\section{Abstract}

Knowledge on the mechanical behavior of the implant system is an important parameter in predicting a prosthetic rehabilitation. Objective: To assess the influence of bone tissue shape on stress distribution in Morse taper implant and bone tissue by the method of three-dimensional finite elements. Materials and method: Three model shapes were used for bone tissues: a) parallelepiped, b) elliptical, and c) generated from tomography data. Implants were installed in the three-dimensional models, receiving the application of two loads of $100 \mathrm{~N}$ in the craniocaudal direction: 1) vertically along the central axis; and 2) oblique at $45^{\circ}$ from the central axis, from buccal to lingual aspect. Results: The maximum Von Mises stresses (MPa) in models of Morse taper implant and parallelepiped bone tissue were 14.30 in vertical loading and 60.78 in oblique loading. In the elliptical bone tissue model, stress was 14.69 in vertical loading and 71.66 in oblique loading. In the bone tissue model originated from the tomography, stress was 15.04 in vertical loading and 79.15 in oblique loading. Conclusion: The shape of bone tissue models had an influence on stress behavior and distribution, and the model generated from tomography data showed the highest stress values.

Keywords: Dental implants. Finite elements analysis. Mechanics.

\section{Referências}

1. Branemark PI, George AZ, Albrektsson T. Tissue-integrated prostheses: osseointegration in clinical dentistry. Chicago: Quintessence; 1985. 612p.

2. Albrektsson T. Osseointegrated oral implants: a swedish multicenter study of 8139 consecutively inserted nobelpharma implants. J Dent Res 1988; 59(5):287-88.

3. Kan B, Coskunses FM, Mutlu I, Ugur L, Meral DG. Effects of inter-implant distance and implant length on the response to frontal traumatic force of two anterior implants in an atrophic mandible: threedimensional finite element analysis. Int J Oral Maxillofac Surg 2015; 44(7):908-13.

4. Topkaya T, Solmaz MY. The effect of implant number and position on the stress behavior of mandibular implant retained overdentures: Athree-dimensional finite element analysis. J Biomech 2015 July 16; 48(10):2102-9.

5. Dixon DL. Comparison of screw loosening, rotation, and deflection among three implant designs. J Prosthet Dent 1995; 74(3):270-8.

6. Geng JP. Application of finite element analysis in implant dentistry: a review of the literature. J Prosthet Dent 2001; 85:585-98.

7. Rangert B. Bending overload and implant fracture: a retrospective clinical analysis. Int J Oral Maxillofac Implants 1995; 10(3):326-34.

8. Shibata Y, Tanimoto Y, Maruyama N, Nagakura M. A review of improved fixation methods for dental implants. Part II: Biomechanical integrity at bone-implant interface. J Prosthodont Res 2015; 59(2):84-95.

9. Verri FR, Santiago Júnior JF, Almeida DA, Verri AC, de Souza Batista VE, Lemos CA, et al. Three-dimensional finite element analysis of anterior single implant-supported prostheses with different bone anchorages. Scien Wo Journal 2015; 2015:321-28 
10. Mehdi G, Belarbi A, Mansouri B, Azari Z. Numerical study of effect of elastomeric stress absorbers on stress reduction in bone-dental implant interface. J Appl Oral Sci 2015; 23(1):87-93.

11. Rubo JH, Souza EAC. Métodos computacionais aplicados à bioengenharia: soluções de problemas de carregamento em próteses sobre implantes. Rev Fac Odontol Bauru 2001; 9(3/4):97-103.

12. Çehreli MC, Akça K, Iplikçioglu H. Force transmission of one and two piece Morse-taper oral implants; a nonlinear finite element analysis. Clin Oral Implants Res 2004; 15:481-9.

13. Bulaqi HA, Mousavi Mashhadi M, Safari H, Samandari MM, Geramipanah F. Effect of increased crown height on stress distribution in short dental implant components and their surrounding bone: A finite element analysis. J Prosthet Dent $2015 ; 113(6): 548-57$.

14. Costa C, Peixinho N, Silva JP, Carvalho S. Study and characterization of the crest module design: A 3D finite element analysis. J Prosthet Dent 2015; 113(6):541-7.

15. Garitaonaindia U, Alcaraz JL. Influence of a micro-thread at cervical position and a cylindrical intermediate zone on the mechanical behaviour of dental implants: A three-dimensional finite element analysis. Proc Inst Mech Eng H 2015; 229(9):670-80.

16. Alves Filho A. Elementos finitos: a base da tecnologia CAE. São Paulo: Érica; 2000. 292p.

17. Kitamura E, Stegaroiu R, Nomura S, Miyakawa O. Biomechanical aspects of marginal bone resorption around osseointegrated implants: considerations based on a three-dimensional finite element analysis. Clin Oral Implants Res 2004; 15(4):401-12

18. Sutpideler M. Finite element analysis of effect of prosthesis height, angle of force application, and implant offset on supporting bone. Int J Oral Maxillofac Implants 2004; 19(6):819-25

19. Holmes DC, Loftus JT. Influence of bone quality on stress distribution for endosseous implants. J Oral Implantol 1997; 23(3):104-11

20. Akagawa Y. A mimic osseointegrated implant model for three-dimensional finite elemet analysis. J Oral Rehabil 2003 ; 30:41-5.

21. Lekholm U, Zarb GA. Pacient selection y preparcion. In: Branemark PI, Zarb GA, Albrektsson T. Protesis Tejido. Integradas La oseointegracion em La odontologia clínica. Chicago: Quintessence; 1985.

22. Chun HJ. Evaluation of design parameters of osseointegrated dental implants using finite element analysis. J Oral Rehabil 2002; 29:565-74.

23. Huang HL. Biomechanics of splinted/ non-splinted crowns supported by various implant designs. J Dental Res 2004; 83:707-14.

24. Ishigaki S. Biomechanical stress in boné surrounding na implant under simulated chewing. Clin Oral Implant Res 2003; 14:97-102.

25. Lin TS. The effect of retainer thickness on posterior resin-banded prostheses: a finite element study. J Oral Rehabil 2004 ; 31:1123-9.

26. Weinstein AM, Allan M. Stress analysis of porous rooted dental implants. J Dent Res 1976; 55:772-7.

27. Akça K, Iplikçioglu H. Finite element stress analysis of the effect of short implant usage in place of cantilever extensions in mandibular posterior edentulismo. J Oral Rehabil 2002; 29:350-6.
28. Dalkiz M, Zor M, Aykul H, Toparli M, Aksoy S. The three-dimensional finite element analysis of fixed bridge restoration supported by the combination of teeth and osseointegrated implants. Implant Dent 2002; 11(3):293-300.

29. Jaffin RA, Berman CL. The excessive loss of Branemark fixtures in type IV bone: a 5 year analysis. J Periodontol 1991; 62(1):2-4.

30. Clift SE, Fisher J, Watson CJ. Finite element stress and strain analysis of the bone surrounding a dental implant: effect of variations in bone modulus. Proc Inst Mech Eng 1992; 206(4):233-41.

31. Hutton JE. Factors related to succes and failure rates at 3-year follow-up in a multicenter study of overdentures supported by Branemark implants. Int J Oral Maxillofac 1995; 10(1):33-42

32. Alexander H, Weiner S, Zweig B, Simon J, Ricci JL. FEA prediction of crestal boné retention around dental implants. J Dent Res 2004; 83:52-72.

33. Haraldson T, Carlsson GE. Bite force and oral function in patients with osseointegrated oral implants. Scand J Dent Res 1977; 85:200-8.

34. Morgan MJ, James DF. Force and moment distributions among osseointegrated dentals implants. J Biomech 1995; 28(9):1103-9.

35. de Castro Albuquerque R, Polleto LT, Fontana RH, Cimini CA. Stress analysis of an upper central incisor restored with different posts. J Oral Rehabil 2003; 30:936-43.

36. Jeong JH, Kim JS, Koo TH. Stress analysis of implant prosthesis according to direction of load. J Dent Res 2004; 83:585-98.

37. Kitagawa T, Tanimoto Y, Nemoto K, Aida M. Transient dynamic analysis of dental implants by finite element method. $\mathrm{J}$ Dent Res 2004; 83:219-24.

38. Verri FR, Torcato LB, Pellizer EP. Influence of osseointegrated implant diameter and length associated with RDP. J Dent Res 2004; 83:24-35.

39. Falk H, Laurell L, Lundgren D. Occlusal force pattern in dentitions with mandibular implant-supported fixed cantilever prostheses occluded with complete dentures. Int J Oral Maxillofac Implants 1989; 4(1):55-62.

40. Papavisilou G, Kamposiora P, Bayne SC, Felton DA. 3D-FEA of osseointegration percentages and patterns on implant-bone interfacial stresses. J Dent 1997; 25(6):485-91.

41. Hansson AS. A conical implant-abutment interface at the level of the marginal bone improves the distribution of stresses in the supporting bone. Clin Oral Implants Res 2003; 14:286-93.

Endereço para correspondência:

Estevão Tomomitsu Kimpara

Av. Francisco José Longo, 77

12245-000 São José dos Campos, SP

Telefone: (12) 997457248

E-mail: estevao@fosjc.unesp.br

Recebido: 13/04/16. Aceito: 29/06/16. 